
allemande

51-1 | 2019

La République démocratique allemande dans l'espace public européen (1949-2018)

\title{
Die DDR aus polnischer Perspektive
}

Wahrnehmungen und Erinnerungsdiskurse am Beispiel Preußens in den 80er Jahren

\section{Burkhard Olschowsky}

\section{(2) OpenEdition}

\section{Journals}

Édition électronique

URL : https://journals.openedition.org/allemagne/1570

DOI : $10.4000 /$ allemagne. 1570

ISSN : 2605-7913

Éditeur

Société d'études allemandes

Édition imprimée

Date de publication : 2 juillet 2019

Pagination : 141-154

ISSN : 0035-0974

Référence électronique

Burkhard Olschowsky "Die DDR aus polnischer Perspektive“, Revue d'Allemagne et des pays de langue allemande [Online], 51-1 | 2019, Online erschienen am: 02 Juli 2020, abgerufen am 02 Juni 2022. URL: http://journals.openedition.org/allemagne/1570 ; DOI: https://doi.org/10.4000/allemagne.1570 


\section{Die DDR aus polnischer Perspektive Wahrnehmungen und Erinnerungsdiskurse am Beispiel Preußens in den 80er Jahren}

\section{- Burkhard Olschowsky*}

Die Geschichte Polens und der DDR waren auf spezifische Weise miteinander verflochten. Sie waren unmittelbare Nachbarn im geografischen Sinne, aber auch in ökonomischer und mentaler Hinsicht. Die im Sommer 1945 gezogene Oder-Neiße-Grenze betraf die Deutschen in der DDR in besonderer Weise. Die geografische Nähe, das Kappen traditioneller Handelswege und der Verlust wichtiger Agrarflächen im Osten wirkten sich desaströs auf die ostdeutsche Wirtschaft und Versorgung aus. Zudem strandete die erste Welle von „Umsiedlern“, wie sie offiziell zu heißen hatten, nach sogenannten wilden Vertreibungen bereits im Frühsommer 1945 diesseits der Oder und Neiße. Die SED sträubte sich in den ersten Jahren, die höchst unpopuläre Grenzziehung anzuerkennen, wie sie im Potsdamer Abkommen festgehalten worden war. Moskau intervenierte erst im Zuge des sich anbahnenden Kalten Krieges 1947/1948 und drängte auf die Unterzeichnung des Görlitzer Vertrages über die Oder-NeißeGrenze zwischen der DDR und der Volksrepublik Polen am 6. Juli $1950^{(1)}$.

Mit der Anerkennung setzte die DDR-Regierung einen Standard im bilateralen Verhältnis und den internationalen Beziehungen, an dem sich insbesondere die westdeutschen Regierungen jahrzehntlang messen lassen mussten und hinter den sie in jahrzehntelang intensiv geführten politischen und öffentlichen Debatten - nicht zurückgehen konnten ${ }^{(2)}$. Die polnische Führung musste die DDR fortan als „ersten friedlichen Arbeiter- und Bauernstaat auf deutschem Boden“ im eigenen Land präsentieren. Was selbst bei Anerkennung der Oder-Neiße-Grenze durch die DDR nach den

* Wissenschaftlicher Mitarbeiter im Bundesinstitut für Kultur und Geschichte der Deutschen im östlichen Europa (BKGE).

1 Sheldon Anderson, A Cold War in the Soviet Bloc. Polish-East German Relations 1945-1962, Boulder, Perseus, 2001, S. 35-45.

2 Burkhard Olschowsкy, Oder-Neiße-Grenze, <ome-lexikon.uni-oldenburg.de/begriffe/oder-neissegrenze> (27.01.2019). 
Jahren der deutschen Okkupation und des aufgestauten Hasses gegen alles Deutsche schwer genug war ${ }^{(3)}$.

In der DDR bildete die Abgrenzung von der nationalsozialistischen Vergangenheit das Fundament des von der SED propagierten staatlichen Selbstverständnisses. Die „antifaschistische Tradition" taugte zu der nicht gänzlich erfolglosen propagandistischen Negativkontrastierung der Bundesrepublik ${ }^{(4)}$. An der Seite der „siegreichen Sowjetunion" wurde der ostdeutsche Staat von der SED-Führung von der Verantwortlichkeit für die im deutschen Namen verübten NS-Verbrechen freigesprochen. Die Verantwortung für dieses Kapitel deutscher Geschichte hatte ausschließlich der westdeutsche Staat zu tragen. Eine Haltung, die in Polen, so der Publizist Peter Bender, nicht gerade vertrauenerweckend wirkte ${ }^{(5)}$. Diese abrupte Wandlung, wonach die DDR im Zeichen des Antifaschismus nunmehr die "guten“ Deutschen repräsentierte, wurde in Polen schwerlich geglaubt, auch wenn die Anerkennung der Oder-NeißeGrenze allgemein für gut geheißen wurde. Mehr noch,

„die selektive Haltung der SED-Führung zur deutschen Geschichte verhinderte einen substantiellen deutsch-polnischen Dialog, eine Auseinandersetzung über die politische und moralische Verantwortung der Deutschen und somit über die Konsequenzen, die aus der Geschichte für das zukünftige Verhältnis zu ziehen waren. Ein solches, politischen Erwägungen folgendes ostdeutsches Geschichtsbewusstsein vertiefte eher das Misstrauen zwischen Polen und Deutschen“(6).

Für die Deutschen in der DDR war die Oder-Neiße-Grenze schmerzlich nahe, bis in die 70er Jahre nicht oder nur schwer passierbar und aus dem öffentlichen Diskurs verbannt. Die Gründung landsmannschaftlicher Organisationen blieb den vier Millionen Vertriebenen in der DDR untersagt. Wer die „Oder-Neiße-Friedensgrenze“ kritisierte, lief Gefahr, als Feind des Friedens und Faschist kriminalisiert zu werden. Hierbei handelte es sich um keine spezifisch ostdeutsche Erfahrung. Die Amputation des öffentlichen Bewusstseins und Privatisierung der Erinnerung an Heimat und Vertreibung teilten die Ostdeutschen mit den Polen, die über den Verlust etwa von Lemberg oder Wilna ebenfalls keine Auseinandersetzung führen durften. Der verlorene Osten war mit einem dauerhaften Tabu belegt ${ }^{(7)}$.

Polen und Ostdeutsche erlebten wie der jeweilige Nachbar entgegen allen negativen Erfahrungen zum sozialistischen Bruderland erklärt wurde. Wo authentische Versöhnung von Nöten gewesen wäre, wurden ritualisierte Freundschaftskundgebungen veranstaltet, die die Betroffenen eher verstörten, als füreinander einnahmen. Wenn es Organisationen wie die Aktion Sühnezeichen gab, die sich unter jungen Leuten

3 Frank Bontscheк, Die Volksrepublik Polen und die DDR: Ihre Beziehungen und ihre Probleme, Berichte des Bundesinstituts für ostwissenschaftliche und internationale Studien, Köln, 1975, S. 12.

4 Jurek Becker, „Mein Vater, die Deutschen und ich“, Die Zeit, 20.05.1994, S. 58.

5 Basil Kerski, „Zwangsverordnete Freundschaft? Die Beziehungen zwischen der DDR und Polen. Versuch einer Bilanz", in: Basil Kerski, Die Dynamik der Annäherungen in den deutsch-polnischen Beziehungen. Gegenwart und Geschichte einer Nachbarschaft, Düsseldorf, Düsseldorf university Press, 2011, S. 142-157, hier S. 145; Peter Bender, Unsere Erbschaft. Was war die DDR - was bleibt von ihr?, Hamburg, Luchterhand, 1992, S.93-94.

6 B. Kerski, „Zwangsverordnete Freundschaft?“ (Anm. 5), S. 146.

7 Michael Schwartz, „Vertreibung und Vergangenheitspolitik. Ein Versuch über geteilte deutsche Nachkriegsidentitäten“, Deutschland-Archiv, 30/2 (1997), S. 177-195. 
beiderseits der Grenze für eine authentische Versöhnung einsetzten, wurden insbesondere von der DDR-Führung als überflüssig betrachtet und beargwöhnt ${ }^{(8)}$.

\section{Die DDR-Polen-Beziehungen in den 70er Jahren}

Zur positiven Zäsur der Beziehungen zwischen beiden Gesellschaften wurde die Öffnung der Grenze für den visafreien Reiseverkehr ab dem 1. Januar 1972. Dieser Schritt, ein Novum im damaligen Ostblock, war von Gierek und Honecker als Zeichen der Annäherung beider Länder und als Ende der zwischen Gomułka und Ulbricht gepflegten Animositäten aufgefasst worden. Beiderseits der Oder-Neiße-Grenze nahm die Bevölkerung die Möglichkeit, ungehindert das Nachbarland zu bereisen, mit Freude und Neugier auf. Trotz der Sprachbarriere machten sie sich ein eigenes Bild vom Nachbarland und seinen Bewohnern. Polen avancierte zum wichtigsten Reiseland für die DDR-Bevölkerung. Die Ostdeutschen entkamen auf diese Weise ihrem Eingesperrtsein, für viele Polen, denen wegen fehlender Devisen die Fahrt nach dem „Westen“ unmöglich war, bot die Grenzöffnung gleichfalls die erste Gelegenheit zur Auslandsreise. Millionenfach überquerten Polen und Ostdeutsche jährlich die Grenze, hunderte von betrieblichen Kooperationsbeziehungen und institutionellen Partnerschaften in der Kultur und Wissenschaft wurden in den 70er Jahren geschlossen. Als Folge der vielen Berührungspunkte ergaben sich Freundschaften und etwa 10.000 Ehen ${ }^{(9)}$. Gegen Ende der 70er Jahre wurden die Zollkontrollen der DDR unnachgiebiger und die Verärgerung in den grenznahen Bezirken über die polnischen Käufer größer ${ }^{(10)}$.

Trotz dieser Irritationen war die Ära Gierek die fruchtbarste Dekade in den Beziehungen zwischen Polen und der DDR. Die Reise vieler Ostdeutscher in ehemals deutsche Gebiete mochte den Schmerz über den Verlust der Heimat mildern, zumal die Vertreibung in der DDR tabuisiert war und man sich nur im privaten Kreis äußern konnte. Die Offenheit und Gastfreundlichkeit der Polen wurde weithin geschätzt. Was sich in typischen Kommentaren wie, „in Polen lässt es sich leben“ oder „wir fühlen uns hier wie zu Hause“ ausdrückte. Neben den schönen Landschaften besaßen die Ergebnisse des Investitionsprogrammes, das Angebot einiger Westprodukte, aber auch der im Vergleich zur DDR weniger reglementierte, buntere Alltag und die kulturelle Vielfalt eine nicht geringe Anziehungskraft auf die Ostdeutschen. In Polen trugen die neuen Reisemöglichkeiten zu einer positiven Veränderung des Bildes von den Ostdeutschen bei. Die Belastungen aus der nationalsozialistischen Vergangenheit traten im Fall der DDR zeitweilig hinter die den Deutschen zugeschriebenen Sekundärtugenden wie Ordnung, Arbeitsethos, Genauigkeit und Sauberkeit zurück ${ }^{(11)}$.

8 Konrad WeIß, Lothar Kreyssig. Prophet der Versöhnung, Stuttgart, Bleicher Verlag, 1998, S. 367.

9 Mieczysław Tomala, Patrząc na Niemcy. Od wrogości do porozumienia, Warschau, Polska Fundacja Spraw Międzynarodowych, 1997, S.451-455; Andrzej KwILEcki, „Studies on the Stereotype of the ,German' and the ,Pole' in the GDR and FRG“, Polish Western Affairs, $19 / 2$ (1978), S. 300-301; Franciszek GoŁembowski, „Polska w opinii krajów sąsiednich“, Sprawy Międzynarodowe, 39/4 (1986), S. 147.

10 Januszem Rolicki, Edward Gierek: Przerwana dekada, Warschau, 1990, S. 85, 100; Stiftung Archiv der Parteien und Massenorganisationen der DDR im Bundesarchiv (SAPMO-BA), DY vorl. SED, 31970/1, In den Warenhäusern am Alexanderplatz, in Schwedt, Neubrandenburg, Görlitz und Frankfurt/Oder ging der Umsatz nach Einstellung des visafreien Reiseverkehrs mit Polen am 30.10.1980 durchschnittlich um mehr als $1 / 4$ zurück.

11 A. KWILECKI, „Studies on the Stereotype of the ,German' and the ,Pole' in the GDR and FRG“ (Anm. 9), S. 300-301. 


\section{Preußenrenaissance}

In den 70er Jahren begann die DDR-Führung die Existenz einer „sozialistischen Nation der Arbeiter und Bauern“ für das Land zwischen Oder und Werra zu reklamieren. Mit diesem bloßen Plädoyer ließ sich kein tragfähiger DDR-Patriotismus erzeugen. Die DDR-Bürger verstanden sich in großer Zahl weiterhin auch als Deutsche und Erben einer deutschen nationalen Kultur. Offiziell befördert setzte eine Debatte darüber ein, auf welche Teile sich das Erbe des ostdeutschen Teilstaates stützen konnte. Wobei sich die preußische Geschichte aufgrund der vielfältigen geografischen Bezüge und regionalen Traditionen als zentral erwies.

Historiker der DDR konnten fortan und mit Quellenkenntnis intensiv über Preußen forschen und zur Ausprägung des erweiterten Geschichtsbildes in der DDR beitragen ${ }^{(12)}$. Mit ihren Forschungen hatten sie Einfluss darauf, welche Teile der preuBischen Geschichte für die DDR traditionswürdig und einem historisch interessierten Lesepublikum zugänglich gemacht wurden. 1979 rehabilitierte mit Ingrid Mittenzwei, eine führende Historikerin der Akademie der Wissenschaften der DDR, den Preußenkönig Friedrich II $^{(13)}$.

Sichtbarer Ausdruck der Preußenrenaissance in beiden deutschen Staaten waren die Rückkehr des Reiterstandbildes von Friedrich II. Unter die Linden im Jahre 1980 und die große Ausstellung Preußen - Versuch einer Bilanz 1981 in West-Berlin. Diese beiden Ereignisse verliefen in ihrer öffentlichen Resonanz denkbar verschieden: Die Wiederaufstellung des Reiterstandbildes wurde weder öffentlich aufgeführt noch begründet, sondern insgeheim und ohne öffentliche Diskussion und Erklärung vorgenommen. Die drei Monate währende Preußen-Ausstellung im frisch restaurierten Martin-Gropius-Bau unmittelbar an der Berliner Mauer war ein wohl inszeniertes Ereignis, das breite öffentliche und publizistische Aufmerksamkeit fand ${ }^{(14)}$.

In Polen wurde diese Hinwendung zur preußischen Geschichte durch Historiker wie Journalisten mit Aufmerksamkeit, aber auch Unbehagen wahrgenommen. Das alte Schema wonach Ereignisse oder historischen Jubiläen, die in beiden deutschen Staaten begangen wurden, im Falle der DDR zu rechtfertigen, für die Bundesrepublik dagegen zu verurteilen, stieß Anfang der 80er Jahre an seine Grenzen. Nach über einem Jahrzehnt der Entspannungspolitik glaubten die betreffenden Journalisten kaum mehr an diese künstliche Dichotomie, zudem erlaubten die Monate der Solidarność vom August 1980 bis Dezember 1981 eine Berichterstattung weitgehend frei von Zensurvorgaben ${ }^{(15)}$.

12 Ingrid Mittenzwei, „Die zwei Gesichter Preußens. Über die Haltung zu unseren Traditionen am Beispiel der preußischen Geschichte“, Forum, 19 (1978), S.8-9; Horst BARTEL, „Vorbemerkung“, in: Peter Bachmann, Inge Knотн (Hg.), Preußen - Legende und Wirklichkeit, Berlin (Ost), Dietz, 1983, S. 5-9, hier S. 9.

13 Ingrid Mittenzwei, Friedrich II. von Preußen. Eine Biographie, Berlin, Akademie Verlag, 1979; Jan Herman BRINks, Die DDR - Geschichtswissenschaft auf dem Weg zur deutschen Einheit. Luther, Friedrich II. und Bismarck als Paradigmen politischen Wandels, Frankfurt am Main, Campus, 1992, S.266-268.

14 Preußen. Versuch einer Bilanz, Gottfried Korff (éd.), Ausstellung im Gropius-Bau, 15.08.-15.11.1981, Berlin, 1981.

15 Andreas Lawaty, Das Ende Preußens in polnischer Sicht. Zur Kontinuität negativer Wirkungen der preußischen Geschichte auf die deutsch-polnischen Beziehungen, Berlin/New York, Walter De Gruyter (Veröffentlichungen der Historischen Kommission zu Berlin, 63), 1986, S. 256. 
Alle größeren polnischen Zeitungen widmeten sich dem Thema Preußen. Die Rückkehr des Friedrich-Denkmals an seinen alten Platz Unter den Linden kommentierte Marek Regel spöttisch, wonach es Nationen gebe, unter denen es der einen an Wurst, der anderen wiederum an historischer Verwurzelung mangele ${ }^{(16)}$. Im Parteiorgan Trybuna Ludu dämonisierte Henryk Olszewski Preußen „zum Symbol des Deutschtums, zum Synonym für Stärke und Hoffnung auf Einheit, zum Vorposten aller Teutonen gen Osten, zum Nachfolger der Mission des Deutschen Ritterordens, zur Stütze gegen die neuen Gefahren des Liberalismus, der Demokratie, des Sozialismus“. Am preußischen Ungeist sei die Weimarer Republik gescheitert. „Das Großdeutsche Reich Hitlers wurde zur logischen Nachfolge der Triumphe preußischer Generale.“ Abschließend lässt Olszewski nicht unerwähnt, dass die Preußenwelle auf eine Idealisierung Preußens ziele, um dadurch „die theoretischen Grundlagen für eine Politik der Wiedervereinigung Deutschlands anzureichern“(17).

Die kulturell anspruchsvolle Parteizeitung Polityka widmete dem Thema mehrere Artikel $^{(18)}$. Adam Krzemiński beschrieb seine Eindrücke von den Besuchen im OstBerliner Museum für Deutsche Geschichte und der West-Berliner Preußenausstellung. Beide hinterließen bei ihm einen bitteren Nachgeschmack, weil er im ersten Museum vor der geballten proletarischen Geschichtsdarstellung stand - in der Bismarck zwar ausgiebig im Zusammenhang mit dem Sozialistengesetz, nicht aber mit der Germanisierungspolitik Erwähnung fand - und sich fragte, welches Bild wohl der deutsche Betrachter von der gemeinsamen Nachbarschaft bekommt. In West-Berlin stieß sich Krzemiński an der Darstellung des preußischen Alltags mit all seiner Provinzionalität, die einem die Luft nehme. Schließlich gestand er der DDR das Recht zur eigenen, also auch zur preußischen Geschichte zu, während er hinter der westdeutschen Preußeneuphorie die in seinen Augen abwegige preußische Mission nach einer Wiedervereinigung sieht. Zudem „bemängelte er das Aussparen des polnisch-preußischen Verhältnisses aus den Preußenbildern beider deutscher Staaten, was es deutlich erschwere, jungen Deutschen oder wenig informierten Besuchern die historischen Belastungen des preußisch-polnischen Verhältnisses nahezubringen ${ }^{(19)}$.

Die beiden parteiloyalen Journalisten Ryszard Wojna und Julian Bartosz betrachteten wohl auch aus ideologischen Gründen die Bemühungen um ein erweitertes Erbe wohlwollend. Wojna bediente sich der Argumentation von DDR-Historikern, wonach die DDR-Bevölkerung ein solch hohes "gesellschaftliches Bewusstsein“ erreicht habe, dass man ihr nun auch die preußische Geschichte vermitteln könne. Dem „unvorbereiteten“ polnischen Betrachter müsse dies jedoch, wie Wojna meinte, erklärt werden, selbst wenn diese Bemühungen durchaus vergeblich sein könnten. Abschließend spricht er der deutschen Frage jegliche Aktualität ab, da die DDR - trotz anderslautender Äußerungen aus der Bundesrepublik - unerlässlich sei für die sozialistische Gemeinschaft, die europäische Stabilität und Friedensordnung ${ }^{(20)}$.

16 Marek Regel, „Historia unter den Linden“, Przekrój, 22.03.1981.

17 Henryk Olszewski, „Nostalgia za Prusami“, Trybuna Ludu, 12.06.1981.

18 Adam Krzemiński, „Prusy, Prusy i co dalej?“, Polityka, 29.08.1981; Marek Regel, „Dziesiątki oczy Frederyka Wielkiego“, Polityka, 06.12.1986.

19 A. Krzemiński, „Prusy, Prusy i co dalej?“ (Anm. 18).

20 Ryszard Wojna, „Prawo Niemców do Historii“, Polityka, 17.03.1984. 
Julian Bartosz betrachtete die Differenzen zwischen Polen und der DDR in ihrer Sicht auf Preußen als normal und unproblematisch, da beide Länder durch die „Klassenposition und ihren Internationalismus" unwiderruflich miteinander verbunden seien. Die 35-jährige Existenz der DDR sei das sichtbare Zeichen der europäischen Friedensordnung und Garant für die Abwehr großdeutscher Ambitionen. Das Misstrauen seiner Landsleute gegenüber der preußischen DDR verurteilte Bartosz als ein Charakteristikum polnischer „Spießer“, die unbelehrbar nach historischen Analogien suchten und nicht das qualitativ Neue in Gestalt der DDR erkennen würden ${ }^{(21)}$.

Kontrovers verlief die Diskussion, ob sich die beiden deutschen Staaten durch die Preußenwelle annäherten und so insgeheim auf die Wiedervereinigung zusteuerten. Die einen sprachen der deutschen Frage jegliche Aktualität ab, da die DDR unerlässlich für die sozialistische Gemeinschaft, die europäische Stabilität und Friedensordnung sei. Für andere, wie dem Journalisten Marian Podkowiński, genügte die wohlwollende Besprechung von Ingrid Mittenzwei 1979 verfasster Biographie über Friedrich II. in der Bundesrepublik, um vor Wiedervereinigungsbestrebungen zu warnen ${ }^{(22)}$. Als hinlänglichen Beweis für die Gefahr der Wiedervereinigung machte Podkowiński eine im Westfernsehen ausgestrahlte Defa-Produktion über Scharnhorst aus, zumal diese gute Kritiken - insbesondere das Lob Sebastian Haffners - erhielt. Erleichtert nahm Podkowiński zur Kenntnis, dass die DDR-Geschichtsbücher in ihrer Darstellung noch nicht von der Preußenwelle verändert wurden, wo Preußen also unverändert und ausschließlich als , aggressiver Militärstaat“ dargestellt wurde. Emotionale Ablehnung schob sich hier deutlich vor eine sachliche Beschreibung dessen, was Preußen im Horizont des 17., 18. und 19. Jahrhunderts über die Pickelhaube hinaus auch war ${ }^{(23)}$.

In das Jahr 1981 fiel auch der im Untergrund zirkulierende und in der Bundesrepublik veröffentlichte Aufsatz „Zwei Vaterländer. Zwei Patriotismen“ des Literaturwissenschaftlers und Oppositionellen Jan Józef Lipski. Indem er die Vertreibung von Millionen Deutschen aus den ehemaligen deutschen Ostgebieten als Unrecht und den deutschen „Drang nach Osten“ als Mythos und „Ausrede für uns“ bezeichnete, beging Lipski gleich mehrere Tabubrüche ${ }^{(24)}$. Seine entwaffnende Offenheit löste in der offiziellen Presse aggressive Wut aus. Man warf ihm vor, er würde die Eroberungen Friedrichs des Großen gewissermaßen nachträglich rechtfertigen ${ }^{(25)}$. In der Untergrundpresse fand Lipskis Aufsatz große Aufmerksamkeit aber keineswegs nur Zustimmung. In der Bundesrepublik fühlten sich die Vertriebenenverbände in ihrer Haltung bestätigt, während die liberale Öffentlichkeit das Thema Vertreibung - aus

21 Julian BArtosz, „To dopiero początek“, Sprawy i Ludzie, 04.10.1984. Im gleichen leninistischen Tonfall unterscheidet Bartosz zwischen „guten“ und „bösen“ Deutschen: J. BARTosz, „Który Niemiec jest dobry?", Sprawy i Ludzie, 20.05.1982.

22 Marian Podkowiński, „Czy Nostalgia za Pickelhauba? Motywy tzw. Fali Pruskiej w RFN“, in: Antoni Czubiński (Hg.), Problematyka Prus wczoraj i dziś, Poznań, Wielkopolskie Wydawnictwo Prasowe, 1981, S. 81.

23 M. Podkowiński, „Czy Nostalgia za Pickelhauba?“ (Anm. 22), S. 82.

24 Jan Józef Lipski, „Zwei Vaterländer - zwei Patriotismen. Bemerkungen zum nationalen Größenwahn und zur Xenophobie der Polen“, in: Ders., Wir müssen uns alles sagen..., Warschau, Deutsch-Polnischer Verlag, 1996, S. 185-228.

Rafał ŁĄKowski, „Pour le roi de Prusse“, Argumenty, n 49, 06.12.1981, S. 4. 
falsch verstandener Versöhnungsabsicht - scheute und es mied, Lipskis zivilgesellschaftliche Vorstellungen zur Überwindung der Jalta-Ordnung zu diskutieren ${ }^{(26)}$.

Die populären Vorstellungen wie jene vom Dämon Preußen waren fest verwurzelt, nicht von heute auf morgen aufgegeben wurden. Erschwerend kam hinzu, dass in der deutschen Geschichtsschreibung die in hohem Maße preußische Politik zu Lasten von Polen kaum thematisiert wurde, was zwischen Oder und Bug wiederum in Wissenschaft und Publizistik genau registriert wurde. Derlei Ahnungslosigkeit oder Ignoranz auf deutscher Seite erschwerte es in Polen, das regionale Erbe in seiner Gesamtheit, einschließlich seiner preußischen Hinterlassenschaften in den Nord- und Westgebieten Polens anzunehmen. „Insbesondere Preußens Bauten lagen der polnischen Gesellschaft wie Steine im Magen. Sie waren die unverdaulichen Anteile der 1945 mehr oder weniger oktroyierten Landnahme“(27).

Der scheinbare deutsch-deutsche Gleichklang bei der Hinwendung zur preußischen Geschichte waren mitnichten die Vorboten einer deutschen Wiedervereinigung, sondern für die DDR-Regierung Ausdruck der steten Konkurrenz zum westdeutschen Teilstaat. Der Geschichtswissenschaft als ideologiebildender Faktor kam hierbei die eine besondere Rolle zu. Sie sollte „alle progressiven Traditionen der deutschen Geschichte hervorheben“ und diese „im Kampf gegen den in der BRD vertretenen Antikommunismus und bürgerlichen Nationalismus massenwirksam zur Geltung bringen “(28).

Der Umstand, dass die West-Berliner Preußen-Ausstellung im Gropius-Bau und damit unmittelbar an der Berliner Mauer zu sehen war, nährte die Befürchtung der Genossen in Ost-Berlin auf Verlust über die Deutungshoheit dieses Teils der deutschen Geschichte zumal sie keine eigene Preußen-Ausstellung vorzuweisen hatten, um sich, so die Annahme der SED-Führung, mit den Sichtweisen des „Klassengegners" auseinandersetzen zu können. Was blieb war der Auftrag für das 750-jährige Berlin-Jubiläum im 1987 besser gewappnet zu sein und bis dahin eine eigene ostdeutsche Berlin-Geschichte zu verfassen, nach der sich dann geplante Ausstellungen ausrichten sollten $^{(29)}$.

\section{Polnische und DDR-Historiker über Preußen}

Preußen war ein Thema, dass zur kontroversen und produktiven Beschäftigung der Historiker beider Länder geeignet schien. 1970 fand in Posen eine Konferenz statt, bei der die Ergebnisse der jüngsten polnischen Preußenforschung vorgestellt wurden und auf weitgehende Zustimmung bei den anwesenden DDR-Historikern stießen ${ }^{(30)}$. Zehn Jahre später waren die Historiker beider Länder weniger einmütig, als sie sich

26 Jacek KuвıAк, „Uwagi o stosunku opozycji demokratycznej do zagadnień niemieckich”, in: Anna Wolff-Powęska (Hg.), Polacy wobec Niemców, Poznań, Instytut Zachodni, 1993, S. 397-398.

27 Gregor Thuм, „,Preußen - das sind wir!' Zur Wiederentdeckung der preußischen Kulturlandschaft in Deutschland und Polen“, Zeitschrift für Ostmitteleuropa-Forschung, 59/3 (2010), S. 301-321, hier S. 314.

28 Anne Wanner, „Wie stellt der Klassenfeind die preußische Geschichte aus? Die Wahrnehmung und Wirkung der 1981 in West-Berlin gezeigten Sonderausstellung, Preußen - Versuch einer Bilanz in der DDR“, Deutschland-Archiv, 20.04.2018, S. 1-10.

29 Ebd., S.4-6.

30 Hans Hübner (Halle) und E. Moritz (Halle): Antoni Czubiński, „Problematyka Prus jako przedmiot zainteresowań badawczych historiografii Polskiej Rzeczypospolitej Ludowej“, in: DERs. (Hg.), Problematyka Prus wczoraj i dziś (Anm. 22), S. 15. 
in einer Nummer des Przeglad Zachodni des Posener West-Instituts mit der Preußenwelle beschäftigten. Horst Bartel stellte Friedrich II. innen- und verwaltungspolitisches Reformwerk in den Kontext der marxistischen Geschichtsphilosophie. Ihm sei der Übergang vom „spätfeudalen Militarismus“ Friedrich Wilhelms I. zu den angeblich „frühbürgerlichen“ Reformern Stein, Hardenberg, Scharnhorst und Clausewitz zu verdanken, darum könne Friedrich II. bei aller Kritik an seiner Außenpolitik als „fortschrittlich“ gelten. Bartel wirbt zugleich um Verständnis dafür, daß die DDR als der Staat auf einstigem preußischem Kernland zwangsläufig einen Teil seiner Tradition und seines Erbes aus der preußischen Vergangenheit beziehe ${ }^{(31)}$. In gleicher Richtung wie Bartel argumentierte Ingrid Mittenzwei, die Friedrich II. als aufgeklärten Monarchen mit einem Hang zur Kunst und Wissenschaft unversehens zum Beschützer des schwach ausgeprägten Bürgertums macht ${ }^{(32)}$. Walter Schmidt erklärt die unterschiedlichen Seiten der Politik Friedrich II. „dialektisch“. Die Regierungsjahre Friedrich II. seien „- gegen seinen subjektiven Willen - auf die Stabilisierung der veralteten Feudalherrschaft ausgerichtet. Objektiv setzten sie jedoch Veränderungen in Gang, die zur Überwindung des Feudalismus“ geführt hätten ${ }^{(33)}$.

Gegen die Gruppe genannter DDR-Historiker, die ausnahmslos auf die marxistische Formationstheorie rekurrierten, schrieb der 1981 mit einer Biographie über Friedrich II. aufwartende Thorner Historiker Stanisław Salmonowicz an ${ }^{(34)}$. Er bestritt eine geistige Affinität Friedrich II. für das Bürgertum, es sei stattdessen das bloße merkantilistische Kalkül gewesen, das Friedrich II. mit einzelnen Bürgern verbinde. Die unbestreitbaren Leistungen Friedrichs II. auf dem Weg Preußens zur europäischen Großmacht, basierten auf einem viel zu hohen Preis, den Europas - und vor allem Polens - Bevölkerung für diesen Aufstieg zu zahlen gehabt hätten. Die Kontinuität preußischer "Tugenden“ habe, so Salmonowicz, der deutschen Geschichte einen verhängnisvollen Verlauf mit dem Endpunkt 1945 beschert $^{(35)}$.

Das von DDR-Historikern vorgetragene Argument, es bedürfe allein deshalb der intensiven "materialistisch-dialektischen“ Auseinandersetzung mit der preußischen Geschichte, um dem bundesdeutschen „Preußenkult“ sowie der Vereinnahmung dieses wichtigen Teils deutscher Geschichte begegnen zu können ${ }^{(36)}$, wurde von den

31 Horst Bartel, „W kwestii marksistowsko-leninskiego obrazu Prus w NRD“, Przegląd Zachodni, 4 (1980), S. 30-38.

32 Ingrid Mittenzwei, „Absolutyczne Prusy w historii niemieckiej“, Przegląd Zachodni, 4 (1980), S. 55-60.

33 Walter SснміDт, „Historia narodowa NRD a terytorialno-państwowe dziedzictwo historyczne“, Przeglad Zachodni, 4 (1980), S. 65.

34 Stanisław Salmonowicz, Frederyk II, Wrocław, Ossolineum, 1981. Im gleichen Jahr erlebte das während der deutschen Besatzungszeit geschriebene und 1947 erstmals publizierte Buch von Władysław Konopczyński (1880-1952) Frederyk Wielki a Polska (Poznań) seine zweite(!) Auflage. Konopczyński vertritt die These, dass Preußen seine territoriale und politische Macht nur dadurch ausweiten konnte, weil Polen im Zustand der Machtlosigkeit und Anarchie gehalten wurde.

35 Stanisław Salmonowicz, „Frederyk wielki władcą oświeconego absolutyzmu?“, Przegląd Zachodni, 4 (1980), S. 42, 48-49. Marian Drozdowski führt die negativen ökonomischen Folgen der Teilung von 1775 vor allem für die polnischen Bauern an: Marian Drozdowski, „Osiemnastowieczne źródła antagonizmu między Polską a Prusami“, Przegląd Zachodni, 4 (1980), S.72-87.

36 I. Mittenzwei, „Die zwei Gesichter Preußens“ (Anm. 12); „Den politischen und ideologischen Inspiratoren von systematisch und langfristig angelegten Forschungen wie spektakulären 
meisten polnischen Historikern weder methodisch, noch in seinem Anspruch ernst genommen $^{(37)}$. Der Umstand, dass die preußische Teilungspolitik in den 50er und 60er Jahren noch ausdrücklich als antipolnisch und ausbeuterisch verurteilt worden war, in den späteren Jahren jedoch nur als Teil der Außenpolitik Erwähnung fand, rief bei polnischen Historikern ebenso Beunruhigung hervor wie die Nonchalance bei der geographischen Bezeichnung der eroberten polnischen Gebiete - zum Beispiel war statt Podlasie „Neu-Ostpreußen“ zu lesen $^{(38)}$.

Als eigentlichen Grund für die Missverständnisse zwischen polnischen und deutschen Historikern aus Ost und West machte Klaus Zernack treffend den Mangel aus, Begriff und Reichweite der preußischen Geschichte aus der Wirkung Preußens in der Geschichte der deutsch-polnischen Beziehungen abzuleiten. Er führte die „polonozentrischen Einseitigkeiten“ auf die geringe Rezeptionsbereitschaft in der deutschen Historikerschaft zurück, wodurch die polnischen Historiker mit ihren Arbeiten auf sich selbst zurückverwiesen waren. Deutsche Historiker tendierten dazu, etwa den polnischen Einfluss auf die preußischen Agrargeschichte oder die Herausbildung des deutschen Nationalstaates zu übersehen, während die polnische Geschichtswissenschaft sich nur schwer von einer mitschwingenden Abneigung gegen die politische Kultur des Ordensstaates freimachen konnten. Die republikanische Maiverfassung von 1791 wurde quasi systemideologisch, so Zernack, zur Alternative gegen den monarchischen Absolutismus überhöht, welche dann Preußen, Russland und Österreich zum Kampf gegen den polnischen „Jakobinismus“ geführt habe ${ }^{(39)}$.

Hinsichtlich der Fehlwahrnehmungen der polnischen Geschichtswissenschaft merkte Gerard Labuda bereits 1971 an, dass die in der polnischen Historiographie gängige „Anschauung über die Eroberungssucht des brandenburgisch-preußischen

Schauveranstaltungen geht es letztlich darum, dem imperialistischen Staat BRD ein historisches Fundament zu schaffen und der sozialistischen DDR gewissermaßen nur eine Außenseiterrolle in der deutschen Geschichte zuzuweisen“: Gustav Seeber, „Einleitung. Preußen seit 1789 in der Geschichtsschreibung der DDR“, in: Gustav SeEber, Karl-Heinz NoACK (Hg.), Preußen in der Geschichte nach 1789, Berlin, Akademie Verlag, 1983, S.46; Lozek meint, die westdeutsche Historiographie bemächtigte sich des Themas Preußen, um die „expansionistische These von der angeblichen , nationalen Mission' der BRD historisch abzustützen“: Gerhard LOzEK, „Grundfragen der aktuellen Auseinandersetzung mit der bürgerlichen Historiographie“, Beiträge der Geschichte der Arbeiterbewegung, 22/1 (1980), S.28-29.

37 Der Stellenwert der DDR-Preußenforschung nimmt sich bei Labudas weitem Blick in die Forschungslandschaft gering aus: Gerard LABudA, „Die Geschichte Preussens in den Anschauungen der deutschen und nicht-deutschen Historiographien ", Polnische Weststudien, 5/1 (1986), S. 3-34; Włodzimierz Borodziej und Tadeusz Cegielski analysieren in Polen am ausführlichsten die DDR-Preußenforschung. Die Autoren bemängeln den inflationären und unklaren Gebrauch von Begriffen wie „fortschrittlich“, „reaktionär“, „Revolution von oben“, welcher der marxistischen Geschichtswissenschaft geschuldet ist und die den preußischen Staat mal als „Vermittler zwischen Adel und Bürgertum“ das nächste Mal in Form des „feudalistischen Absolutismus“ beschreibt: Włodzimierz BorodzIEJ, Tadeusz CegIELSKI, „Prusy i tradycja pruska w historiografii NRD“, Kwartalnik Historyczny, 4 (1981), S. 996.

38 Die Literaturangaben bleiben die Autoren für die 50er und 60er Jahre-Literatur hier allerdings schuldig. Ebd., S.999, 1002; Alexander KraUß, Die Rolle Preußens in der DDR-Historiographie, Frankfurt am Main, Peter Lang, 1993, S. 49-50.

39 Klaus Zernack, „Die Geschichte Preußens und das Problem der deutsch-polnischen Beziehungen“, Jahrbücher für die Geschichte Osteuropas, 31/1 (1983), S.30, 35-36, 38-40; siehe die inhaltliche Auseinandersetzung Zernacks mit dem Aufsatz von Marian Biskup im gleichen Band der Jahrbücher: Marian Biskup, „Preußen und Polen Grundlinien und Reflexionen“, Jahrbücher für die Geschichte Osteuropas, 31/1 (1983), S. 1-27. 
Staates in richtigen Proportionen dargestellt wird. Man darf die eigene Unfähigkeit nicht ausschließlich den Eroberern zur Last legen“(40). Die Preußen-Renaissance in beiden deutschen Staaten Anfang der 80er Jahre ließ Labudas Plädoyer ungehört verhallen. Ein Teil der mit Preußen befassten polnischen Historiker sah sich in der Auffassung bestätigt, als Korrektiv zur vermeintlichen (west-)deutschen „Preußenglorifizierung" die preußische Germanisierungspolitik weiterhin akzentuieren zu müs$\operatorname{sen}^{(41)}$. An diesem Unterfangen hatte, wie oben dargelegt, die polnische Publizistik einen großen und nicht selten ungestüm vorgetragenen Anteil.

\section{Erweiterung des polnischen Traditionsreservoirs}

Ähnlich wie die DDR Friedrich II. in ihren Traditionsreservoir aufnahm, verfuhr Polen in den 80er Jahren mit Józef Piłsudski, dessen Verdienste um die Wiedererlangung der polnischen Unabhängigkeit nun auch die Trybuna Ludu nicht mehr schmälern mochte ${ }^{(42)}$. Polens Unabhängigkeit in der Zwischenkriegszeit wurde als positive Errungenschaft per se dargestellt. Im Unterschied zur DDR hatte der historiographische Diskurs jedoch weit weniger mit der offiziellen Politik zu tun. Anders ausgedrückt, die Grenzen wissenschaftlicher Autonomie waren um vieles weiter gesteckt als in der DDR. Historiker wie Bogdan Suchodolski und Ryszard Ludwikowski analysierten die (politische) Kultur der zweiten Republik und deren Einfluss auf das Handeln der politischen Akteure, ohne sich einer klassenbezogenen Sozialgeschichte verpflichtet zu fühlen ${ }^{(43)}$.

Die Politiker taten das ihre. Aus Anlass des 60. Jahrestages der Unabhängigkeit hielt Edward Gierek eine Rede im Sejm, in der er sich vorsichtig dem Thema näherte und dabei die Anerkennung der jungen polnischen Republik durch die Bolschewiki sowie die Teilnahme „vieler Tausend Polen an der Oktoberrevolution“ rühmte. Der polnisch-sowjetische Krieg blieb ein $\mathrm{Tabu}^{(44)}$. Die neue Parteilinie erklärend, meldeten sich Ryszard Wojna sowie der ZK-Sekretär und Direktor des „Instituts für Marxismus-Leninismus beim ZK der PVAP“, Andrzej Werblan, zu Wort ${ }^{(45)}$. Wojna erhob die Volksrepublik Polen zum moralischen und politischen Erben einer tausendjährigen Tradition mit dem Schlüsseljahr 1918, in dem Polens moderne Staatlichkeit entstand. Werblan sah mit der Entstehung der zweiten Republik die nationale Frage gelöst, die jedoch erst nach 1944 in der Verbindung mit der sozialen Frage Polens Unabhängigkeit und Entwicklung dauerhaft gesichert habe ${ }^{(46)}$.

40 Gerhard Labuda, „Rewizja dziejów Prus - osiągnięcia i plany badań“, in: Ders. (Hg.), Węzłowe problemy dziejów Prus XVII-XX wieku?, Poznań, Instytut Zachodni, 1971, S. 10.

41 A. Czubiński, „Problematyka Prus jako przedmiot zainteresowań badawczych historiografii Polskiej Rzeczypospolitej Ludowej“(Anm. 30), S. 7-22.

42 Michał Berezowski, „11 Listopada - W rocznicę odzyskania niepodległości“, Trybuna Ludu, 11.11.1981.

43 Bogdan Suchodolski, „Polskie 60-lecie - Fakty historyczne i świadomość narodowa“, in: Halina Jankowska, Maria Nowak-KıєŁвıкowa (Hg.), Z perspektywy sześćdziesięciu lat, Warschau, Państwowy Instytut Wydawniczy, 1982, S. 147-168, hier S. 147; Ryszard Ludwikowski w dyskusji, „Kultura polityczna w II Rzeczypospolitej“, Dzieje Najnowsze, 12/1 (1980), S.95, 112.

44 Harald LAeuen, „60 Jahre Wiederherstellung Polens“, Osteuropa, 29/7 (1979), S. 553.

45 Ryszard Wojna, „Sixty years after“, Polish Perspectives, 21/7-8 (1978), S. 9-15; Andrzej Wer BLAN, „Polska Ludowa - Urzeczywistnienie celów społecznych i narodowych“, Z pola walki, 22/4 (1979), S.3-19. 
1981 wurde erstmals seit 1938 der 11. November als Unabhängigkeitstag offiziell gefeiert. Der Vorsitzende des Staatsrates, Henryk Jabłoński, legte an diesem Tag in Begleitung von Marian Orzechowski, einen Kranz am Grabmal des Unbekannten Soldaten nieder ${ }^{(47)}$. Dass beide Historiker waren, mag die Symbolik dieses Aktes unterstreichen. Für die polnischen Kommunisten hatte die nationale Frage nun Vorrang vor der sozialen bei der Bewertung der II. Republik, obwohl diese sich in wenigen Jahren von einer parlamentarischen Demokratie zu einer Obristendiktatur gewandelt hatte. Die Wiederaneignung des Piłsudski-Erbes geschah mit einer Selbstverständlichkeit, die es in der DDR beim Umgang mit Friedrich II. nicht gab. Die Kritik aus Polen hatte ihren Anteil daran. Jabłoński, von Hause aus Geschichtsprofessor, hatte sich 1981 im Gespräch mit Hans-Dietrich Genscher über die Preußen-Renaissance beklagt. Als dieser ihm bedeutete, dass er die Preußen-Renaissance positiv sehe, entgegnete Jabłoński: „Ich meine ja gar nicht die BRD“(48).

\section{Fazit}

Nach dem Zweiten Weltkrieg unter dem Eindruck der deutschen Besatzung 1939 bis 1945 dominierte in Polen die Überzeugung, dass der Nationalsozialismus aus der deutschen, mithin der preußischen Geschichte herrühre. Etwaige positive Entwicklungen des deutschen Volkes wurden durch den preußischen Einfluss verbogen und entstellt. Im kollektiven Bewusstsein vieler Polen geriet Preußen zur schwarzen Legende mit langanhaltender Virulenz. Begünstigend wirkten dabei das jahrzehntelange integrierende Einvernehmen zwischen Staat und Gesellschaft in Polen in der Erinnerung an deutsche Kriegsverbrechen und der Kampf gegen die Okkupanten aus dem Westen ${ }^{(49)}$.

Die Betrachtung Deutschlands durch das Prisma Preußen bzw. das Stereotyp Preußen blieb auch in den weiteren Jahrzehnten der Volksrepublik zentral. Die schwarze Preußenlegende spiegelte sich auch in der offiziell propagierten und lange von der Bevölkerung als alternativlos erlebten Überzeugung wieder, in den West- und Nordterritorien habe der polnische Staat 1945 „urpolnisches“ Land wiedergewonnen, in dem folglich die fremden preußisch-deutschen Spuren zu tilgen und die Oder-NeißeGrenze zu verteidigen war.

Nach 1970 und der de facto-Anerkennung von Polens Westgrenze durch die Bundesrepublik veränderte sich durch eigene positive Erfahrungen zu Deutschen aus der DDR wie aus der BRD das durch den Krieg belastete Deutschlandbild schrittweise. Diese Veränderungen, obgleich vergleichsweise oberflächlich, beeinflussten allmählich das stereotype Bild etwa vom (west)deutschen Vertriebenen der „sein Haus und Hof “ zurückverlangte.

47 Trybuna Ludu, 12.11.1981.

48 Gespräch zwischen Genscher und Schönherr am 16.07.1981, Anlage 117, Anlagen zum Bericht des Stolpe-Untersuchungsausschusses des Landtages von Brandenburg, Potsdam 29.04.1994.

49 Edmund Dmitrów, „Schwarze Preußenlegende“, in: Basil Kerski (Hg.), Preußen - Erbe und Erinnerung, Deutschen Kulturforum östliches Europa, Potsdam, 2005, S. 123-138, hier S. 123-124; Edmund Dмiтrów, Niemcy i Okupacja Hitlerowska w Oczach Polaków z lat 1945-1948, Warschau, Czytelnik, 1987, S. 289-300. 
Mit der Existenz der freien Gewerkschaft Solidarność 1980/81 und dem anschließenden Kriegsrecht kam es in der polnischen Bewertung der beiden deutschen Staaten zu einer spürbaren Akzentverschiebung zugunsten der Bundesrepublik und zu Lasten der DDR. Dies war einerseits nachvollziehbar, da die DDR-Führung jene freie Gewerkschaft als Gefahr betrachtete und diese mit allen politischen Mitteln versuchte einzudämmen. Andererseits nahmen etliche Polen fälschlicherweise an, die die DDRBevölkerung unterscheide sich in ihrer Abneigung gegenüber der Solidarność kaum von den DDR-Machthabern ${ }^{(50)}$.

Der ostdeutsche Teilstaat mit seinem Grenzregime wurde als künstlich wahrgenommen und mit einer Bevölkerung, die in hohem Maße obrigkeitshörig und für den Kommunismus anfällig sei. Verhaltensweisen, welche dem „militärischen Drill“ und der „seelenlosen Disziplin“ Preußens ähnlich schienen und mehr noch, die negativen deutschen, preußischen Traditionen fortsetzten. Die angenommene Übereinstimmung von Regime und Gesellschaft in der DDR äußerte sich u.a. in dem Stereotyp der „roten Preußen“(51).

Auch der Untergrundpresse - vor allem jener Teil der der Endecja-Tradition ${ }^{(52)}$ verpflichtet fühlte - war das Bild der „roten Preußen“, die in ihrem „künstlichen“ Staat obrigkeitshörig und für den Kommunismus anfällig seien, präsent. Die Abneigung gipfelte in Charakterisierungen der Untergrundpresse wie „Pickelhaubendemokratie mit sibirischen Ohrenschützern“"(53).

In einer von der DDR-Botschaft in Warschau 1987 erstellten Analyse zählen die Autoren die positiven und negativen Seiten „zum DDR-Bild in der VR Polen“ auf. Zu den positiven - wenn auch nicht mehr vorrangigen - Charakteristika im DDR-Bild der Polen zählte, dass die DDR ein verlässlicher Verbündeter sei, der Revanchismus und Militarismus ehrlich ablehne und die polnischen Nachkriegsgrenzen voll anerkenne. Bei den negativen Haltungen spielen ökonomische oder ordnungspolitische Erwägungen keine Rolle, vielmehr werden bestimmte Stereotype von kultureller Prägekraft artikuliert. Der Begriff „Deutsche“ wird hier in negativer Weise synonym für DDR-Bürger verwandt.

„Es ist eine nationale Eigenart der Deutschen, dass sie sich nur in einem streng reglementierten Staatswesen wohlfühlen. Die Deutschen sind zu Disziplin, Ordnung und Fleiß sowie zur Achtung der Staatsmacht erzogen“(54).

Die innerdeutschen Beziehungen wurden kritisch betrachtet.

50 Burkhard Olschowsкy, Einvernehmen und Konflikt. Das Verhältnis zwischen der DDR und der Volksrepublik Polen 1980-1989, Osnabrück, Fibre, 2005, S. 101-110.

51 Hans Henning HAHN, „Stereotypen in der Geschichte und Geschichte im Stereotyp“, in: Historische Stereotypenforschung. Methodische Überlegungen und empirische Befunde, Oldenburg, BIS, 1995, S. 190-204.

52 Endecja steht als Abkürzung für die Nationaldemokraten der Zwischenkriegszeit, die mit einer germanophoben Außen- und antisemitischen Innenpolitik aufwarteten.

53 Martin Mühleck, Das Bild der Sowjetunion und Deutschlands in der oppositionellen Publizistik Polens 1976-1989, Magisterarbeit, Humboldt-Universität, 2000, S. 75-79; Piotr LEWICKI, „Demokracja w pickielhaubie z syberyjskimi nausznikami“, Spotkania, 26 (1984), S. 61.

54 Archiv des Bundesbeauftragten für die Unterlagen des Staatssicherheitsdienstes der ehemaligen Deutschen Demokratischen Republik (BStU) MfS HA II/10/57, Bl. 15. 
„Die Politik der Friedenssicherung, der Vernunft und des Realismus, der friedlichen Koexistenz der DDR gegenüber der BRD wird von diesen Kräften meist unter dem Blickwinkel des Strebens nach Wiedervereinigung unter Umgehung der Bündnisverpflichtungen der DDR gegenüber Polen und den anderen sozialistischen Bruderländern dargestellt“(55).

An anderer Stelle hieß es:

„Der DDR wird vorgeworfen, die preußische Politik und Traditionen zu verherrlichen, die polnischen Erfahrungen zu übergehen und ihre eigenen nationalen Interessen ausschließlich zu verfolgen. Vor allem vom antisozialistischen Gegner wird dem hinzugefügt, dass die ,Gemeinsamkeiten in den Auffassungen der DDR und der BRD zu Preußen' ein Beweis für das tief verwurzelte Streben der Deutschen nach Wiedervereinigung sei, das von keinem Polen unterschätzt werden dürfe“(56).

Die am Beispiel von Preußen skizzierten Wahrnehmungs- und Reaktionsmuster zeigen, dass Polen dann zur Intervention bei deutschen historischen Debatten neigten, wenn sie das Geschichtsverständnis und mithin die eigene Identität gefährdet wähnten. Diese Interventionsoption war (und ist) eine weitgehend polnische, das heißt, sie wird Deutschen im umgekehrten Fall nur widerstrebend zugebilligt. Hinter dieser Haltung verbirgt sich ein elementares Misstrauen, ob die Deutschen mit der Last ihrer Geschichte umgehen können, und der Verdacht, sie würden ihre Geschichte auch zu Lasten des Nachbarn umschreiben wollen ${ }^{(57)}$.

Was die Haltung der DDR-Bevölkerung zum Thema Preußen betraf, so vermochte diese mit Hilfe der Preußen-Renaissance ein souveräneres Verhältnis zur Territorialgeschichte gewinnen. Die Vergangenheit im Guten wie im Schlechten als die eigene annehmen, sich also nicht länger als Vollstrecker ausschließlich der positiven Linie zu sehen, während die negativen Ereignisketten verdrängt wurden. Diese neue Akzentsetzung lief darauf hinaus, die nationale Geschichte als integratives Potential und lehrendes Beispiel zu nutzen. In diesen Punkten befand sich die DDR gegenüber den anderen sozialistischen Ländern in einer benachteiligten Lage. Ein gelassenes und differenziertes Urteil über die Vergangenheit erweiterte die historische Basis und erschloss neue Quellen, die das Handeln der Ostdeutschen motivieren konnte. Erst die kritische Auseinandersetzung mit dem preußischen Mythos als einem Ordnungsprinzip, welches Untertänigkeit zum Preis für soziale und ökonomische Sicherheit sowie obrigkeitsstaatliche Fürsorge erhob, mochte den Blick auf die eigene Unmündigkeit in der DDR durchaus schärfen.

In Polen bedurfte es der politischen Wende von 1989, dass ein unvoreingenommenes Verhältnis zum regionalen, mithin preußischen Erbe in den Nord- und Westgebieten auch in der Breite der polnischen Gesellschaft zum Tragen kommen konnte.

55 BStU MfS HA II/10/57, Bl. 15.

56 BStU MfS HA II/10/57, Bl. 16.

57 Diese Erfahrung machte z.B. der Regisseur Frank Beyer, als sein Film Der Aufenthalt, der deutsche Kriegsgefangene in Polen zeigt, auf polnische Intervention 1983 als DDR-Beitrag von der „Berlinale“ genommen wurde; Helga Hirsch stieß mit bei der Präsentation ihres Buches Die Rache der Opfer. Deutsche in polnischen Lagern 1944-1950 (Rowohlt, 1998) auf starke Vorbehalte in Polen. Adam Krzemiński, „Die schwierige deutsch-polnische Vergangenheitspolitik“, Aus Politik und Zeitgeschichte, 40-41 (2003), S. 4 . 


\section{Zusammenfassung}

Die DDR stand in den 80er Jahren verstärkt unter Wiedervereinigungsverdacht als zeitgleich in beiden deutschen Staaten eine Preußenwelle einsetzte, die mit der Ausstellung Preußen - Der Versuch einer Bilanz im West-Berliner Martin-Gropius-Bau und mit der Rückkehr des Reiterstandbildes von Friedrich II. Unter die Linden ihre symbolischen Höhepunkte fand. Die ost- und westdeutsche Preußenwelle nährte in Teilen der polnischen Publizistik die Meinung, die Deutschen betrieben zum Nachteil Polens ihre Annäherung mit dem Ziel der Wiedervereinigung. Dem überdehnten Argument der DDR-Historiker, Friedrich II. sei als Wegbereiter frühbürgerlicher Reformen durchaus "fortschrittlich" gewesen, fand Polens Leserschaft wenig überzeugend. Der Ordensstaat und mehr noch das Preußische in der deutschen Geschichte erfüllten das eindeutig negative Klischee einer Macht, die verhängnisvoll für Deutschlands Nachbarn sei. Mit dieser Haltung bestritt man in Polen der DDR, das zu tun, was für andere sozialistische Länder Normalität war und Polen seit Mitte der 70er Jahre tat - nämlich die Vergangenheit als integratives Potential für die eigene Gesellschaft zu nutzen.

\section{Résumé}

Dans les années 1980, tandis que grandissait en Pologne la crainte de voir renaître la perspective de la réunification, les deux États allemands connurent au même moment un regain d'intérêt pour l'héritage prussien, qui culmina avec l'exposition Preußen der Versuch einer Bilanz au Martin Gropius Bau à Berlin-Ouest et le retour de la statue équestre de Frédéric II sur l'avenue Unter den Linden. Cette "vague prussienne» à l'Est et à l'Ouest nourrit dans les médias polonais l'opinion selon laquelle les Allemands opéreraient un rapprochement aux dépens des Polonais dans le but de parvenir à la réunification. L'argument ressassé par les historiens est-allemands, selon lequel Frédéric II, à travers les premières réformes qu'il initia en matière civile, fut "progressiste", ne rencontra que peu d'écho auprès du lectorat polonais. L'Ordensstaat prussien, et plus encore l'esprit prussien à travers l'histoire allemande confirmaient le cliché strictement négatif d'une puissance dangereuse pour les voisins de l'Allemagne. Ainsi, on refusait à la RDA le droit de faire ce qui constituait une normalité pour d'autres pays socialistes et ce que pratiquait la Pologne depuis le milieu des années 1970, à savoir utiliser le potentiel du passé comme moyen de consolidation sociale.

\footnotetext{
Abstract

In the 80s the GDR was increasingly under Polish suspicion to be engaged for reunification as at the same time in both German states set up a Prussia revival, which had their symbolic highlights with the exhibition Preußen - Der Versuch einer Bilanz in West Berlin's "Martin-Gropius-Bau“ and the return of the equestrian statue of Frederick II "Unter den Linden" in East Berlin. The East and West German Prussian revival nourished in parts of Polish journalism the opinion that the Germans operated to the detriment of Poland their rapprochement with the goal of reunification. The overdrawn argument of the GDR historians that Frederick II had been quite "progressive" as a pioneer of early bourgeois reforms, Poland's readership found hardly convincing. The state of the Teutonic Order, and even more the Prussian part in German history, stimulated the clearly negative stereotype of a power that was disastrous for Germany's eastern neighbors. With this attitude one denied in Poland the GDR, to do what was normal for other socialist countries and Poland did since the mid of 70 s - namely to use the past as an integrative potential for the own society.
} 